\title{
Rhizoctonia spp. Recovered from Strawberry Roots in Central Coastal California
}

\author{
Frank N. Martin
}

U.S. Department of Agriculture-Agriculture Research Service, 1636 East Alisal St., Salinas, CA 93905. Accepted for publication 4 January 2000.

\begin{abstract}
Martin, F. N. 2000. Rhizoctonia spp. recovered from strawberry roots in central coastal California. Phytopathology 90:345-353.

Rhizoctonia spp. were commonly recovered from the roots of strawberry plants growing in nonfumigated soil in the central coastal region of California. With the exception of one multinucleate isolate of $R$. solani (frequency of recovery of $0.8 \%$ ), all other isolates were binucleate and were in anastomosis groups (AG) A, G, or I. AGs-A and -I were recovered from all five collection sites, whereas AG-G was recovered from only two sites. AG-A was the most commonly isolated AG, followed by AGs-I and -G. Similar levels of virulence were observed among the different AGs, but differences in virulence were observed among isolates

mosed with other members of the same AG. Subsequent investigations with multiple isolates in the same AG from the same collection location confirmed that there was a lack of anastomosis or weak anastomosis reactions for some combinations of pairings, highlighting the need for to use multiple tester isolates or molecular techniques for AG determination. Restriction fragment length polymorphism (RFLP) analysis of a polymerase chain reaction-amplified region of the rDNA was effective for differentiating AGs. Sixteen RFLP groups were observed after cluster analysis with data for the size of the amplified products and fragment sizes after digestion with four restriction enzymes. Although each AG had isolates in multiple RFLP groups, any one individual RFLP group contained isolates of only a single AG. There was no consistent correlation between RFLP group and location of isolate collection.
\end{abstract} in the same AG. Evaluating anastomosis grouping by pairing isolates recovered from strawberry with known tester isolates did not always yield a positive anastomosis reaction, even though both isolates anasto-
Additional keywords: binucleate Rhizoctonia spp.
In California, strawberry (Fragaria $\times$ ananassa Duchesne) is cultivated as an annual crop on more than 9,713 ha, was ranked as the number 11 agricultural crop in 1998 with a value of $\$ 783$ million, and represents $76 \%$ of the nation's total strawberry production (1). One of the primary production areas is in the central coastal region of the state encompassing Monterey and Santa Cruz counties, which accounted for $50 \%$ of the states production in 1997 . With the exception of the small percentage of the total strawberry acreage devoted to organic production, virtually all production was done on land that was fumigated with methyl bromide and chloropicrin to control root diseases, nematodes, and weeds $(6,9,18,20-24,26)$. In addition to controlling lethal diseases, such as Verticillium wilt, soil fumigation also controls a number of nonlethal soilborne fungal pathogens that also contribute to significant losses when crops are grown in nonfumigated fields. One disease complex often associated with plants grown in nonfumigated soil is referred to as black root rot. Although not currently a widespread problem in properly fumigated commercial production fields, with the impending phaseout of the use of methyl bromide and alteration of current fumigation practices, this disease complex once again may become a problem.

In other strawberry-production areas in the world, black root rot has been attributed to various combinations of root infection by Pythium spp., three anastomosis groups (AG) of binucleate Rhizoctonia spp., Cylindrocarpon sp., and the lesion nematode Pratylenchus penetrans (25). In Connecticut, it is believed that binucleate

Corresponding author: F. N. Martin; E-mail address: fmartin@asrr.arsusda.gov

Publication no. P-2000-0217-02R

This article is in the public domain and not copyrightable. It may be freely reprinted with customary crediting of the source. The American Phytopathological Society, 2000.
Rhizoctonia spp. and the lesion nematode are primarily responsible for the disease complex (8). In the strawberry-production areas of central coastal California, all of these fungal pathogens have been recovered from necrotic roots of plants growing in poorly fumigated or nonfumigated soil (22,26; F. N. Martin, unpublished data). However, very little is known about the specific contribution of the different pathogens to disease expression or about the different AGs of the binucleate Rhizoctonia spp. that may be present in the local strawberry-production system. The report by Wilhelm et al. (22) on binucleate Rhizoctonia spp. infecting strawberry in California was done prior to the development of the AG classification scheme for these pathogens; therefore, information on the distribution of specific AGs in this production area are lacking.

In Connecticut, three AGs of binucleate Rhizoctonia spp. were recovered from strawberry roots and are believed to be associated with the disease complexes AGs-A, -G, and -I. These AGs vary in their host range among vegetable crops grown in rotation with strawberry (19), as well as the temperature optima for disease development. For example, of the isolates recovered from strawberry in Connecticut, AG-I was reported to be more virulent on strawberry at 10 to $15^{\circ} \mathrm{C}$ than other AGs, whereas AG-G was more virulent than the other two at $24^{\circ} \mathrm{C}(8,12)$. This temperature effect on disease development also appears to influence the time of the year when the respective AGs were commonly recovered from roots; AG-I during the fall (November to December) and AG-G later during the spring (May) (12). Although multinucleate isolates of $R$. solani in AG 5 also were recovered from diseased roots, it was infrequent (12).

Traditionally the classification of AGs of binucleate Rhizoctonia spp. is based on the interaction of an unknown isolate with a known tester isolate. Using isolates from North America, Burpee et al. (2) classified binucleate isolates into seven AGs (CAG1 through CAG7), whereas Ogoshi et al. $(14,15)$ grouped Japanese isolates 
into 17 AGs (AGs-A through -Q), three of which were pathogenic on strawberry. The classification of Ogoshi et al. (14) includes the AGs described by Burpee et al. (2) that are pathogenic on strawberry (CAG-2). The classification of isolates to AG can be a timeconsuming procedure, especially when trying to classify large numbers of isolates recovered from the field. In an attempt to simplify the identification procedure for binucleate Rhizoctonia spp., Cubeta et al. (4) reported on a polymerase chain reaction (PCR)-based procedure in which a region of the $25 \mathrm{~S}$ rDNA is amplified and digested with a variety of restriction enzymes for restriction fragment length polymorphism (RFLP) analysis. The resulting banding patterns correlated well with grouping for a number of AGs, including those commonly recovered from strawberry.

The purpose of this investigation was to evaluate the frequency of recovery of multinucleate and binucleate Rhizoctonia spp. from strawberry roots in the central coastal production area of California. The isolates were classified to $A G$ and evaluated for virulence on strawberry. In addition, the PCR-based procedure for assessment of anastomosis grouping as described by Cubeta et al. (4) was evaluated in the hope that its use will simplify the classification of isolates recovered from the field. It is anticipated that knowledge of the types of Rhizoctonia spp. that are present in the strawberry-production area, their AG classification, and virulence on strawberry will facilitate an understanding of their contribution to the root rot complex infecting strawberry and aid in the development of control procedures.

\section{MATERIALS AND METHODS}

Fungal isolation. Strawberry roots were recovered from commercial plantings growing in nonfumigated soil from different areas of the central coastal production area of California at various times during the 1996 through 1998 seasons. Root samples were collected by either recovering five or more intact plants from the field or collecting multiple soil samples that contained strawberry roots from immediately adjacent to the plant crown. The isolate identification numbers are indicative of the collection location in California and time of the year of recovery for the Watsonville site (B, 2/19/96; C, 3/25/96; D, 4/19/96; 97NF, 2/5/97; and 98M, 3/23/98), Moss Landing site (98sOrt, 4/20/96), Santa Cruz site (98SW, 3/20/98), Salinas site (Sp, 1/30/97; 98Sp, 9/17/98), and Santa Maria site (SM, 3/20/96). All collection sites were cropped with strawberry cv. Selva, with the exception of the Santa Maria location, which was planted with an experimental University of California cultivar.

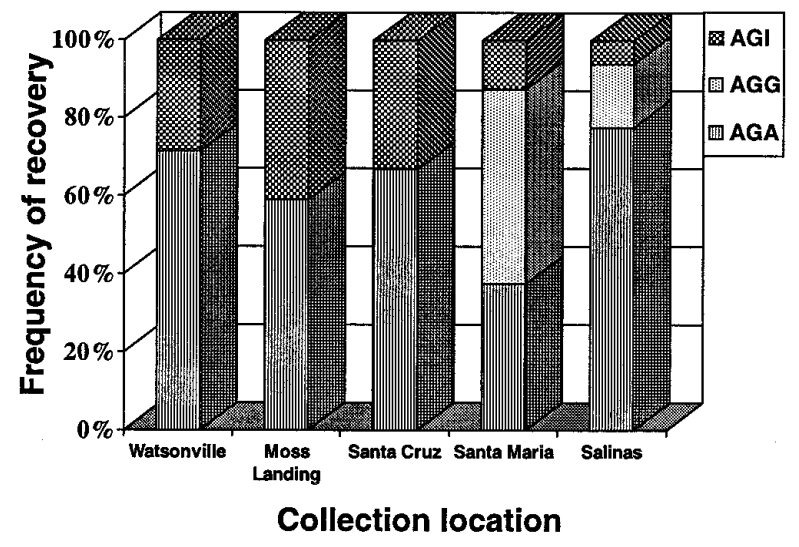

Fig. 1. Frequency of recovery of different anastomosis groups (AG) of binucleate Rhizoctonia spp. from the roots of cv. Selva strawberry plants grown in nonfumigated soil in the central coastal production area of California. The total number of isolates examined from the Watsonville, Moss Landing, Santa Cruz, Santa Maria, and Salinas collection sites were 37, 17, 6, 8, and 51, respectively.
Immediately after collection, the samples were brought back to the lab, and the roots were washed free of soil on a $0.5-\mathrm{mm}$ sieve. Root pieces were blotted dry on paper towels and plated on $1.5 \%$ water agar amended with $0.1 \%$ Tergitol NP-10. Roots $(\approx 200 \mathrm{~cm}$ or more) for each plant were plated on a minimum of eight petri plates. The plates were examined after 24 and $48 \mathrm{~h}$, and colonies thought to be Rhizoctonia or Pythium spp. were transferred to fresh water agar and potato dextrose agar (PDA) plates. Cultures of Rhizoctonia spp. were stored on PDA slants covered with mineral oil or at $-80^{\circ} \mathrm{C}$ using the cryostorage procedure of M. Cubeta (personal communication). Briefly, five $8-\mathrm{mm}$ plugs were removed form the advancing edge of a colony growing on PDA and placed in a cryovial containing $0.5 \mathrm{ml}$ of half-strength potato dextrose broth (PDB). After $24 \mathrm{~h}$ of growth at room temperature, $0.5 \mathrm{ml}$ of $50 \%$ glycerol was added to the cryovial and mixed well. Vials were placed at $-80^{\circ} \mathrm{C}$ for long-term storage.

Determination of AG. Rhizoctonia spp. recovered from roots were examined to determine the number of nuclei per cell by a modification of the procedure of Martin (11). Cultures were grown in half-strength PDB for 2 to 4 days prior to removing a portion of the hyphae and placing it in a drop of water on a glass slide. One drop of bisbenzimide $(5 \mu \mathrm{g} / \mathrm{ml}$; Sigma Chemical Company, St. Louis) was added and mixed with the mycelium prior to covering with a glass coverslip. After $10 \mathrm{~s}$, the dye was removed by placing a paper towel on one side of the coverslip and adding a drop of water on the other side. The slide was viewed under a fluorescence microscope, and the number of nuclei per cell was determined.

Testing for AG was done by transferring agar blocks containing mycelium from the advancing edge of a colony to a thinly poured water agar plate and pairing it with a known tester isolate. Tester isolates of Ogoshi et al. $(14,15)$ for AGs-A, -G, and -I were C-662, C-653, and AV-2, respectively, and were provided by M. Cubeta. Additional tester isolates for AGs-C, -D, -E, -J, -K, -L, -N, -P, -R, and $-\mathrm{S}$ were included in the pairings as well. Cultures were observed several times a day until the hyphae came into contact, at which time the area was covered with a 22-mm coverslip, and a transect of the zone of contact was examined under a compound microscope to determine whether there was anastomosis between different cultures. If one to five hyphal fusions were observed, the anastomosis reaction was classified as weak to intermediate; if more than five fusions were observed, the reaction was classified as frequent. In pairings in which there was no or weak anastomosis, the zone of contact was reexamined later in the day to confirm the observation.

Pathogenicity tests. Selection of isolates to include in pathogenicity tests was based on the level of disease observed in preliminary pathogenicity tests conducted with tall fescue seed inoculum. Briefly, autoclaved tall fescue seeds were placed on water-agar plates individually colonized by a binucleate isolate and allowed to incubate at room temperature for 4 to 7 days. Rooted plantlets produced as described below were transplanted into autoclaved soil and five colonized seeds were placed below the crown among the roots. After 3 weeks of incubation in a growth chamber, under conditions described below, the roots were rated for level of infection. A subgroup of isolates exhibiting the range of symptoms observed in these preliminary trials was selected to include in more detailed, replicated pathogenicity tests with defined amounts of oat-bran inoculum added to the soil.

Inoculum for the more detailed pathogenicity tests was produced by growing isolates in a 500-ml flask containing $134 \mathrm{~g}$ of oat bran and $80 \mathrm{ml}$ of water, similar to the procedure of Mazzola (13). The flasks were autoclaved, inoculated with several agar plug transfers of a specific isolate, and incubated at $25^{\circ} \mathrm{C}$. After 1 week of incubation, the contents of the flask were mixed well with a sterile rod, and after 2 weeks of growth at $25^{\circ} \mathrm{C}$, the culture was dried for several days in a laminar flow hood. After the inoculum was completely dry, it was ground with a mortar and pestle and was passed through a nested 0.85 - and $1.7-\mathrm{mm}$ sieve; the fraction re- 
tained on the 1.7-mm sieve was used as inoculum for the pathogenicity trials.

The soil used for the pathogenicity tests was an Elder sandy loam collected from a strawberry test plot in Watsonville, CA. After collection, the soil was dried and passed through a 5-mm sieve to remove plant material and large clods of soil. To eliminate indigenous pathogens, the soil was pasteurized in an electric sterilizer (Pro-Grow Supply Corp., Brookfield, WI) set at $82^{\circ} \mathrm{C}$ for $2 \mathrm{~h}$ the day before amending with pathogen inoculum. Pathogen inoculum was added on at $0.75 \%$ (wt/wt) for the general pathogenicity screen and at $0.1,0.4$, and $0.75 \%$ for virulence determinations. Infested soil was mixed well prior to placing into $10-\mathrm{cm}$ square pots with 10 pot replicates for each inoculum level tested.

All plant material used for the trials was cv. Selva, which was propagated from runner plants in the greenhouse to ensure that the plants were pathogen free. Mother plants were grown in $15-\mathrm{cm}$ pots in the greenhouse with a wire mesh stretched between the pots and resting on the pot lip. As runners emerged, they were trained to grow on the wire mesh to prevent contact with the bench tops or soil in the mother plant pots. Plantlets were removed from the runners as soon as root initials were visible and stored at $4^{\circ} \mathrm{C}$ in a plastic bag until needed. All plantlets were rooted in autoclaved soil on a misting bench for 2 weeks prior to rinsing the soil off the roots and transplanting into test soils. Pots were transferred into growth chambers with 12-h diurnal changes in lighting, beginning with the lights on for $11 \mathrm{~h}$ at $20^{\circ} \mathrm{C}$; lights off for a 1-h ramp from 20 to $10^{\circ} \mathrm{C}$ and for $11 \mathrm{~h}$ at $10^{\circ} \mathrm{C}$; and lights on for a 1-h ramp from 10 to $20^{\circ} \mathrm{C}$. To control foliar diseases, the plants were sprayed with myclobutanil (Rally 40WP, $0.075 \mathrm{~g} /$ liter) prior to placing in the growth chamber.

Plants were grown for 5 weeks, after which they were removed to determine plant-growth parameters and visual observation of root health. The tops of the plants were cut off to determine fresh weights, and roots were recovered from soil by gentle wet sieving. Roots were examined for disease symptoms, cut off from the crowns, and placed in paper bags for drying at $50^{\circ} \mathrm{C}$ in a forced-air oven for several days prior to weighing. All pathogenicity trials were repeated at least twice. Analysis of variance and liner regression of fresh shoot weights, dry root weights, and incidence of plant death were done using SigmaStat 2.0 (Jandel Scientific Software, San Rafael, CA). For experiments in which the effects of different levels of inoculum on disease severity were tested, the results of regression analysis for each individual isolate were compared with Levene's test for heterogeneity of variance using SAS System for Windows (release 7.00; SAS Institute, Cary, NC).

RFLP analysis. A boiling miniprep procedure that was developed for Pythium spp. was used for isolation of DNA from binucleate isolates for PCR analysis (10). The isolate was grown on PDA plates, and a small amount of aerial mycelium was removed with a sterile transfer needle and placed into a $1.5-\mathrm{ml}$ microfuge tube containing $0.5 \mathrm{ml}$ of sterile water. The tube was incubated in a boiling water bath for 5 min prior to placing the tube at $-20^{\circ} \mathrm{C}$ for long-term storage.

Templates for RFLP analysis were amplified by PCR with primers LROR (dACCCGCTGAACTTAAGC) and LR7 (dTACTACCACCAAGATCT) of Cubeta et al. (4). Amplification reactions were done in $50 \mu \mathrm{l}$ and contained $15 \mu \mathrm{l}$ of boiling miniprep DNA, $25 \mu \mathrm{M}$ each primer, $20 \mu \mathrm{l}$ of $10 \times$ buffer, $100 \mu \mathrm{M}$ each dNTP, $2 \mathrm{mM}$ $\mathrm{MgCl}_{2}$, and $4 \mathrm{U}$ of $\mathrm{Taq}$ polymerase (Promega Crop., Madison, WI). Amplifications were done with a Mastercycler gradient thermocycler (Eppendorf, Westbury, NY) with the following run parameters: 1 cycle at $95^{\circ} \mathrm{C}$ for $2 \mathrm{~min} ; 35$ cycles of 1 -min annealing at $52^{\circ} \mathrm{C}$, 2 min of extension at $72^{\circ} \mathrm{C}$, and $1 \mathrm{~min}$ of denaturation at $94^{\circ} \mathrm{C}$; followed by 1 cycle of extension for $10 \mathrm{~min}$ at $72^{\circ} \mathrm{C}$. For some preparations of DNA, the template did not amplify; dilution of the sample with additional sterile water usually rectified the problem.

After amplification, an aliquot of the reaction mixture was run on a $0.7 \%$ SeaKem agarose gel (FMC Corporation, Rockland, ME) to determine the size and concentration of the amplified fragment or fragments. Subsamples were digested individually with $C f o \mathrm{I}, M s p \mathrm{I}$, Sau3A1, and TaqI in a total volume of $15 \mu \mathrm{l}$ in accordance with the manufacturer's recommendations (Promega). Individual digests were separated on a $3 \%$ Nusieve $3: 1$ gel (FMC) followed by staining with ethidium bromide (17) and photographing of the gel with Polaroid type-55 film. Molecular size markers were 25- and 100-bp ladders (Gibco BRL, Rockville, MD, and New England Biolabs, Beverly, MA, respectively). To simplify comparisons among different gels, several isolates with known RFLP patterns and a range in digested fragment sizes were included in each gel.

To place isolates in groups that reflected their similarities in RFLP banding patterns, cluster analysis was done, using the unweighted pair group method with arithmetic averages (UPGMA). The size of the initial amplified fragments and the products of their digestion were determined, and a data matrix was constructed for each isolate, with 0 representing the absence of a band and 1 representing the presence of a band. UPGMA analysis was done on this data matrix using PAUP 4.02b (Sinauer Associates, Sunderland, MA). A total of 37 character states was included in the analysis.

\section{RESULTS}

Recovery of Rhizoctonia spp. A total of 122 isolates of binucleate Rhizoctonia spp. were recovered from strawberry roots over a period of 3 years. In all, 119 isolates were recovered from plants growing in nonfumigated soil, 1 isolate was recovered from a fumigated Salinas production field (98Miller6), and 2 isolates were recovered from bare-rooted transplants prior to planting in the field (N-1 and BB-1). The recovery of multinucleate $R$. solani was infrequent and restricted to a single isolate from a second-year strawberry crop in Moss Landing.

In addition to Rhizoctonia spp., Pythium spp. were commonly recovered from strawberry roots. When comparing the isolation frequency of these two genera of root pathogens, a total of $30 \%$ of the isolates from the Watsonville site, $61 \%$ from the Moss Landing site, $12 \%$ from the Santa Cruz site, and $50 \%$ from the Salinas site were Rhizoctonia spp. Two other collection sites in the Salinas area were examined as well, but Rhizoctonia spp. were not recovered from these locations. In contrast to these collection locations, the predominant pathogen from the Santa Maria site was Rhizoctonia spp.; very little recovery of Pythium spp. was observed. The time of the season may have had some effect on the differential recovery of the two genera of root pathogens. At the Salinas site, there were frequent isolations of Pythium spp. but not Rhizoctonia spp., from roots collected on 18 March 1998; however, 83\% of the isolations made from roots collected from the same location at the end of the season (17 September 1998) were binucleate Rhizoctonia spp. There was a similar low recovery of binucleate Rhizoctonia isolates from this site on 30 January 1997.

The symptomology of root infection was similar for both pathogens, and they commonly were recovered from necrotic areas of

TABLE 1. Frequency of anastomosis reactions between paired isolates of anastomosis group A binucleate Rhizoctonia spp. from restriction fragment length polymorphism group $\mathrm{O}$, collected from a site in Watsonville, $\mathrm{CA}^{\mathrm{a}}$

\begin{tabular}{lcccccccc}
\hline & \multicolumn{7}{c}{ Isolate } \\
\cline { 2 - 9 } Isolate & D 14 & D 15 & M 18 & M 19 & M 30 & M 36 & M 37 & M 48 \\
\hline D 14 & + & + & - & - & - & + & + & ++ \\
D 15 & $\ldots$ & + & - & + & + & + & + & ++ \\
M 18 & $\ldots$ & $\ldots$ & + & ++ & + & ++ & ++ & + \\
M 19 & $\ldots$ & $\ldots$ & $\ldots$ & + & + & + & + & + \\
M 30 & $\ldots$ & $\ldots$ & $\ldots$ & $\ldots$ & + & + & + & + \\
M 36 & $\ldots$ & $\ldots$ & $\ldots$ & $\ldots$ & $\ldots$ & + & - & + \\
M 37 & $\ldots$ & $\ldots$ & $\ldots$ & $\ldots$ & $\ldots$ & $\ldots$ & + & + \\
M 48 & $\ldots$ & $\ldots$ & $\ldots$ & $\ldots$ & $\ldots$ & $\ldots$ & & + \\
\hline
\end{tabular}

a Frequency of anastomosis between isolates: $-=$ no anastomosis, $+=$ weak to intermediate anastomosis, and $++=$ frequent anastomosis. 
feeder and structural roots. However, infection by Rhizoctonia spp. generally was associated with darker reddish brown necroses than those observed with Pythium spp.

Anastomosis grouping of isolates. Only three AGs of binucleate Rhizoctonia spp., AGs-A, -G, and -I, were recovered. The most commonly isolated AG was A (68\%), followed by AG-I $(21.3 \%)$ and AG-G (10.7\%), but the relative rates of recovery of each AG varied at the different collection sites (Fig. 1). For example, AG-G was recovered from only the Santa Maria and Salinas site, whereas AGs-A and -I were recovered from all sites. At the Watsonville, Moss Landing, and Salinas collection sites, AG-A was the most prevalent AG recovered, followed by AG-I at Watsonville and Moss Landing and AG-G at Salinas. The number of isolates evaluated from Santa Maria and Santa Cruz (eight and six, respec-

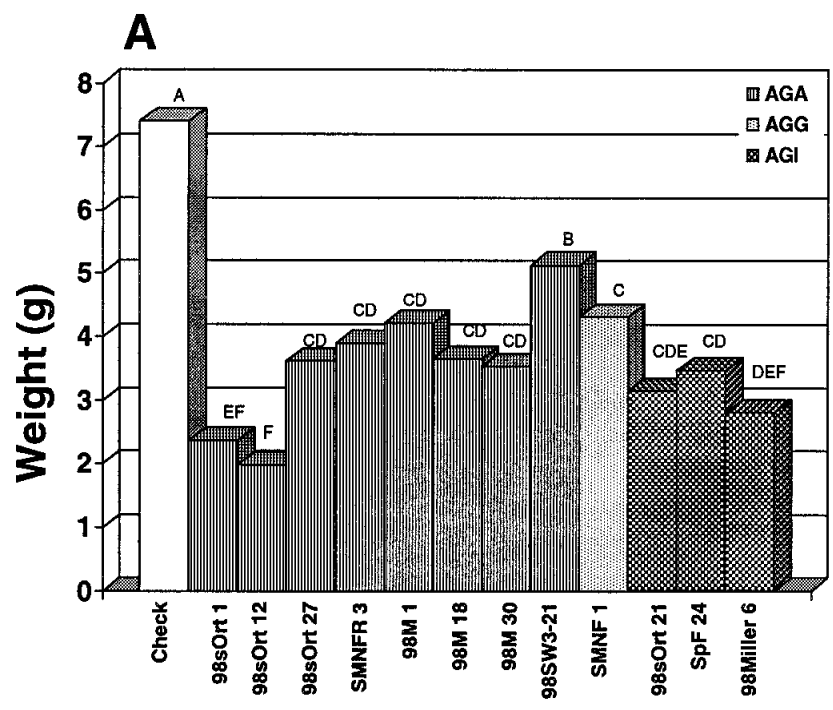

Rhizoctonia spp. isolate

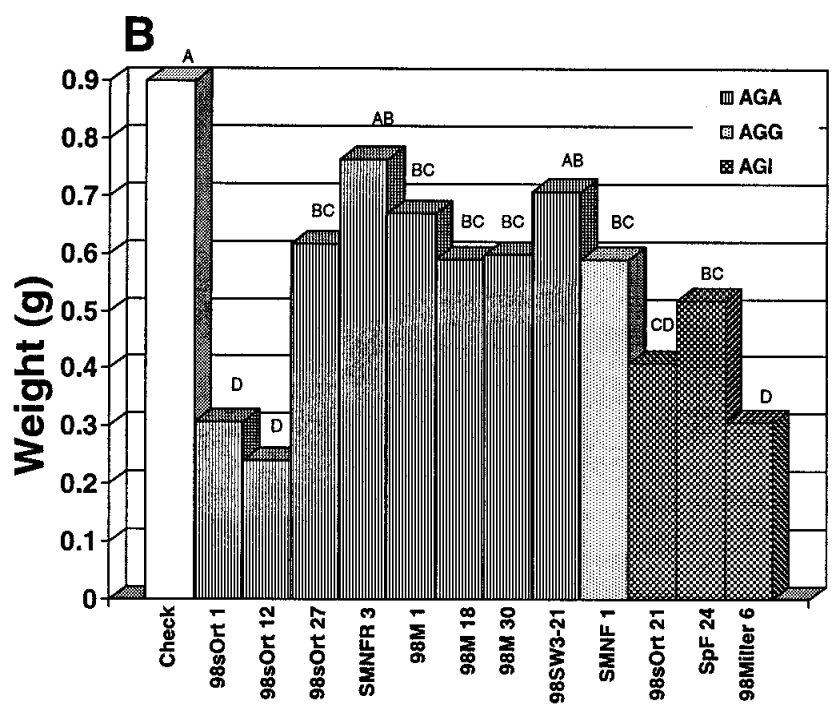

Rhizoctonia spp. isolate

Fig. 2. Influence of different isolates of binucleate Rhizoctonia spp. added to soil at a rate of $0.75 \%$ (wt/wt) on $\mathbf{A}$, fresh shoot weight and $\mathbf{B}$, dry root weight of cv. Selva strawberry plants incubated in a growth chamber for 5 weeks, with 12-h diurnal changes in lighting consisting of lights on for $11 \mathrm{~h}$ at $20^{\circ} \mathrm{C}$; lights off for a 1 -h ramp from 20 to $10^{\circ} \mathrm{C}$ and for $11 \mathrm{~h}$ at $10^{\circ} \mathrm{C}$; and lights on for a 1 -h ramp from 10 to $20^{\circ} \mathrm{C}$. Bars topped by the same letters were not significantly different $(P=0.05)$ according to analysis of variance using the Student-Newman-Keuls test. AG = anastomosis group. tively) that were assessed for AG were too small to accurately evaluate differences in frequency of recovery among AGs.

During the course of evaluating the anastomosis grouping, a number of isolates were observed that did not form a positive anastomosis reaction with any of the three standard tester isolates, yet the RFLP analysis described below suggested that the isolates were AG-A, -G, or -I (data not shown). Subsequent pairings with other strawberry isolates in these three AGs yielded positive anastomosis reactions, indicating that the tester isolates were not effective in identifying the AG designations for all strawberry isolates. To evaluate the extent of the incompatibility of anastomosis reactions among strawberry isolates in the same AG, a subgroup of isolates in RFLP group $\mathrm{O}$ were paired in all possible combinations, and the strength of the anastomosis reaction was evaluated (Table 1). All isolates varied in the frequency of the hyphal anastomosis that was observed in the different pairings, with the strength of the reaction ranging from the lack of anastomosis to frequent anastomosis. Similar results also were obtained when subgroups of isolates in RFLP groups A, D, J, and N were paired among themselves; some pairings among isolates of the same AG did not result in anastomosis between isolates; with others, it was commonly observed (data not shown).

Pathogenicity trials. Preliminary pathogenicity trials with tall fescue seed inoculum revealed that there was a range of root rot symptoms observed among isolates collected during the course of the study (data not shown). A subgroup of isolates reflecting this range in symptom expression was selected for more detailed pathogenicity trials with oat bran inoculum. Addition of inoculum of binucleate isolates of Rhizoctonia spp. to pasteurized soil at a rate of $0.75 \%$ led to significantly reduced shoot weights of strawberry plants for all isolates tested (Fig. 2A). Although there were no consistent differences among AGs, different levels of shoot growth reduction were observed within an AG. For example, in AG-A, there were three different groupings for severity of shoot-growth reductions, with isolates 98 sOrt 1 and 98 sOrt 12 causing the greatest reduction (from 68 to $73 \%$ ), followed by the group containing isolates 98sOrt 27, SMNF3, 98M 1, 98M 18, and 98M 30 (from 43 to $52 \%$ ). Among the AG-A isolates, isolate 98SW3-21 had the least effect on shoot growth (31\% growth reduction). The three AG-I isolates evaluated in this trial had an effect on shoot growth similar to each other and the AG-A and -G isolates; however, inhibition of shoot growth with these isolates was greater than that observed for AG-A isolate 98SW3-21.

These isolates had a similar effect on root growth, albeit to a lesser extent than observed for inhibition of shoot growth (Fig. 2B). Some isolates, such as SMNFR-3 and 98SW3-21, had no significant effect on root biomass when compared with the untreated control plants, whereas all other isolates examined caused significant reductions. Among the AG-A isolates there were only two groupings, with 98sOrt 1 and 98sOrt 12 causing the greatest reduction of root growth (66 to $73 \%$ ), followed by the other AG-A isolates (15 to $35 \%$ weight reduction). As with reductions in shoot growth, the AG-G and -I isolates reduced shoot growth to a similar extent as observed for the AG-A isolates.

Evaluation of isolate virulence by adding varying levels of pathogen inoculum revealed that there were differences in virulence among the isolates examined (Fig. 3). There was a greater reduction in shoot growth per unit of inoculum for isolates 98Miller 6, $\mathrm{SpF} 24$, and 98sOrt 12 compared with the noninoculated control than for the other two isolates, which is exemplified by these more virulent isolates having a steeper slope for the regression lines than the milder isolates 98M18 and 98SW3-21 (-114.2, -93.6, and -81.7 versus -34.1 and -11.5 , respectively; Fig. 3A). Based on extrapolation from the regression analysis, plants inoculated with these isolates at the $0.75 \%$ level had $9.2,29.5,40.4,77.1$, or $88.0 \%$, respectively, of the shoot growth of the untreated control plants. Similar results were observed for root biomass as well, with isolates 98Miller 6, $\mathrm{SpF} 24$, and 98sOrt 12 causing a greater reduction 
in growth per unit of inoculum than isolates 98M18 and 98SW321 (Fig. 3B). Based on extrapolation from the regression line, plants inoculated with these isolates at the $0.75 \%$ level had 9.2, 31.1, $52.4,85.7$, or $82.4 \%$, respectively, of the root growth of the untreated control plants. Statistical comparison of regression slopes for the different isolates revealed that the slopes describing the effect of isolates 98M18 and 98SW3-21 on fresh shoot weight were not significantly different from zero $(P=0.19$ and $P=0.56$, respectively). Similar results also were observed for dried root weight ( $P=0.67$ and $P=0.59$, respectively). The slope of the regression lines describing the effect of isolates 98Miller 6, SpF24, and 98sOrt 12 on both fresh shoot and dried root weight were significantly different from zero $(P<0.05)$ but, due to the level of variance observed in the data (in part caused by the plant mortality noted below), comparison of slopes among these isolates were not significantly different $(P=0.05)$.

The regression analysis for 98SW3-21 was not significant in the experiment $(P=0.37)$, suggesting perhaps that this isolate was not pathogenic (Fig. 3). However, in other experiments (Fig. 2), low levels of disease were encountered with this isolate, so it has been classified as weakly virulent. In addition to reducing shoot and root growth, isolates 98Miller 6 and $\mathrm{SpF} 24$ also caused 30 and $80 \%$ death, respectively, of the plants in the experiment when present at a $0.75 \%$ inoculum level, where the other isolates did not cause any plant mortality.

The data represented in Figures 2 and 3 reflect single experiments. Although slight variations in the data were observed when the experiments were repeated from two to five times, the general trends of the comparisons among isolates and the conclusions that were drawn were the same.

RFLP analysis. PCR amplification of the target sequences revealed that AGs-A and -I had three different isolate groupings for amplified fragment sizes: $1.4,1.8$, or $1.4+1.8 \mathrm{~kb}$. Occasionally, isolates of the two AGs that had $1.4+1.8-\mathrm{kb}$ amplified fragments also had a fainter 1.6-kb band amplified, but this was inconsistent and was not observed in all amplifications. These bands were not included in the data analysis. In contrast, isolates of AG-G had only a single amplified fragment size of $1.4 \mathrm{~kb}$. After digestion of these amplified fragments with 4 restriction enzymes, a total of 16 RFLP groupings was observed (Table 2, Fig. 4). Although there was some variation in the grouping of isolates in the same AG, any one RFLP group contained only a single AG. For example, AG-A was located in two separate clades. One clade (RFLP groups $\mathrm{C}$ and D) contained isolates that had a single amplified band of $1.4 \mathrm{~kb}$ or two amplified bands of $1.4+1.8 \mathrm{~kb}$ and exhibited more similarity in RFLP patterns to a group of AG-I isolates than to the other AG-A isolates. The second clade for AG-A isolates is subdivided into three separate subclades, the groupings of which reflect the initial sizes of the amplified products. Isolates containing initial amplified fragment sizes of $1.4+1.8 \mathrm{~kb}$ were in RFLP groups L and M; isolates with an amplified fragment size of $1.4 \mathrm{~kb}$ were in RFLP group $\mathrm{N}$; and isolates with an amplified fragment size of $1.8 \mathrm{~kb}$ were in RFLP group O. With the exception of the differences between RFLP groups L and M, the different groupings for this clade can be accounted for by the different number or size of the initial amplified products.

Similar results were observed for isolates in AG-I. Isolates recovered from strawberry with amplified fragment sizes of $1.4 \mathrm{~kb}+$ $1.8 \mathrm{~kb}$ were in RFLP group A, whereas the AG-I tester isolate recovered from an Artemesia sp. was in RFLP group B. These isolates grouped closely to the subgroup of AG-A isolates and were on the same clade as the other AG-I isolates that had only a single 1.4-kb amplified fragment (RFLP groups J and K). Isolates of AG-I that had a single 1.8-kb amplified fragment were located on a separate clade distinctly different from all other isolates examined (RFLP group P). Isolates in AG-G all had a single 1.4-kb amplified fragment and were found in five RFLP groups (E through $\mathrm{J}$ ) on a single subclade grouped adjacent to some AG-I isolates.
There was no consistent correlation between RFLP grouping and collection location. Although all isolates in RFLP groups D, E, F, and $\mathrm{P}$ were recovered from the same collection site, isolates in RFLP groups A, G, H, J, L, M, N, and O were from different collection sites. Isolates in the same AG but different RFLP groups also were recovered from a field site at the same collection time. For example, AG-A isolates from the Moss Landing site (labeled 98sOrt) were found in RFLP groups C, D, and N. Similar results were observed with isolates from Salinas (AG-I isolates labeled 98Sp were in RFLP groups A and J; AG-A isolates were in RFLP groups L, N, and O), Watsonville (AG-A isolates labeled D or 98M were in RFLP groups L and O), and Santa Cruz (AG-A isolates labeled 98SW were in RFLP groups L and N).

\section{DISCUSSION}

Rhizoctonia spp. were often isolated from roots of strawberry plants growing in the central coastal strawberry-production area of
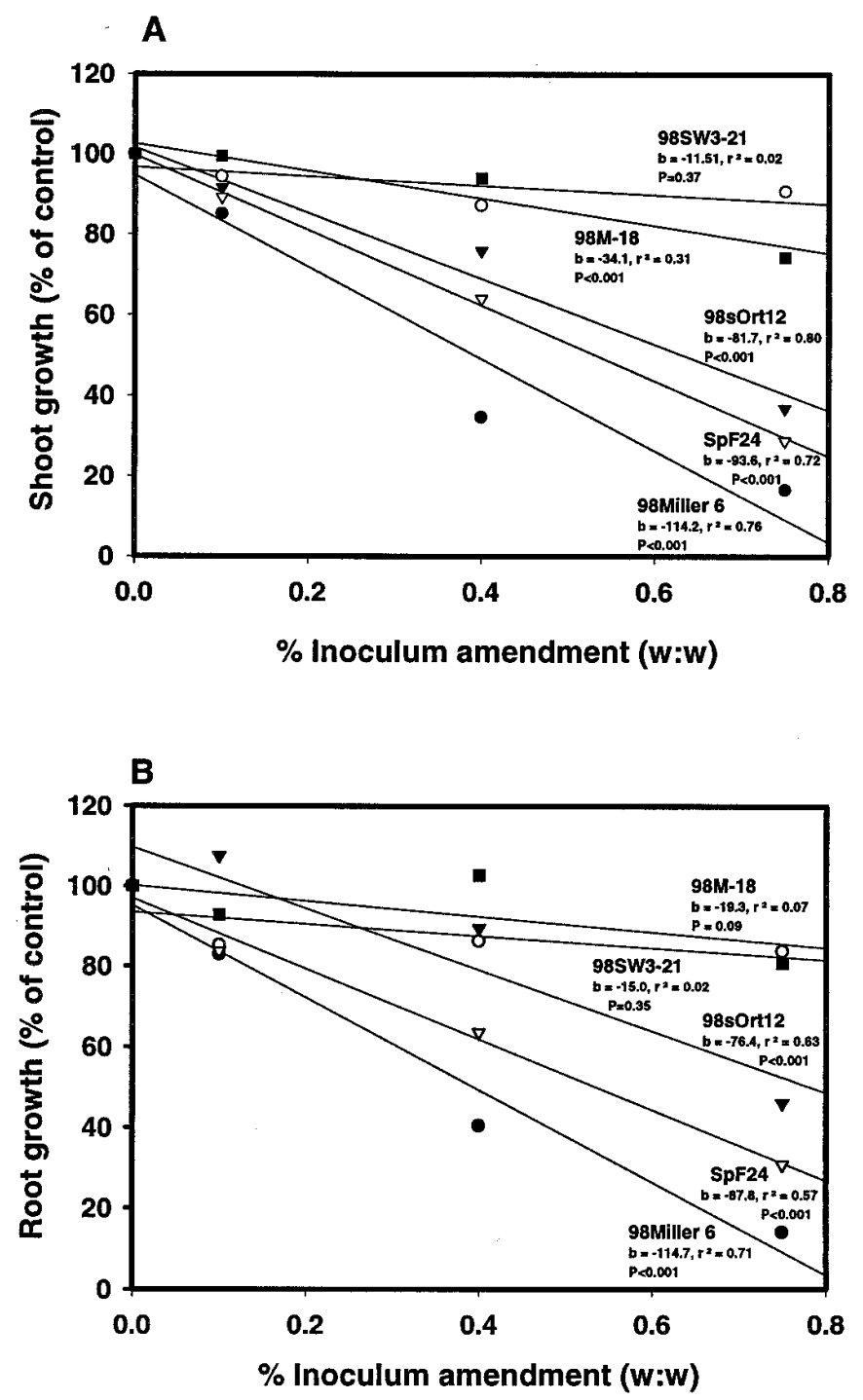

Fig. 3. Linear regression analysis of the influence of inoculum levels of five isolates of binucleate Rhizoctonia spp. on $\mathbf{A}$, fresh shoot weight and $\mathbf{B}$, dry root weight of cv. Selva strawberry plants incubated in a growth chamber for 5 weeks, with 12-h diurnal changes in lighting consisting of lights on for $11 \mathrm{~h}$ at $20^{\circ} \mathrm{C}$; lights off for a 1 -h ramp from 20 to $10^{\circ} \mathrm{C}$ and for $11 \mathrm{~h}$ at $10^{\circ} \mathrm{C}$; and lights on for a 1 -h ramp from 10 to $20^{\circ} \mathrm{C}$. Treatment means for each inoculum level are presented, but linear regression analysis was performed on data for the 10 replicate pots for each treatment; the levels of significance presented on the graph are reflective of this analysis. Isolates evaluated: 98SW3$21(\bigcirc), 98 \mathrm{M}-18(\boldsymbol{\bullet}), 98 \mathrm{sOrt12}(\boldsymbol{\nabla}), \mathrm{SpF} 24(\nabla)$, and 98Miller $6(\bullet)$. 
California. Binucleate Rhizoctonia spp. were the most commonly isolated, with only one isolate of multinucleate $R$. solani recovered from a field containing 2-year-old plants. A total of three AGs of binucleate isolates of Rhizoctonia spp. were recovered: AGs-A, -G, and -I. AGs-A and -I were recovered from all fields examined; however, the distribution of AG-G was more restricted and was present in only two of five locations. The relative frequencies of recovery of specific AGs also varied between collection sites. The results from this survey in California strawberry fields parallels the observations of Martin (12) in surveys taken in Connecticut strawberry-production fields. In his report, there also was an infrequent recovery of multinucleate $R$. solani (less than $3.1 \%$ of the total isolates). The same three AGs of binucleate Rhizoctonia spp. were identified in this study, and their frequency of isolation varied among collection sites. However, a seasonal variation in the frequency of recovery of the different AGs in Connecticut was observed, with AG-G predominating in collections made during the spring (May) and AG-I in collections made during the fall (November to December). The data suggests that there may be a similar seasonal effect on recovery of AGs-I and -G in California. At the Watsonville site, there was a greater recovery of AG-I during the cooler periods of the year (collections B and C) compared with the warmer seasons (collections D and M). Likewise, at the Salinas site, there was a low recovery of binucleate Rhizoctonia spp. during the cooler periods of the year and a high recovery as the seasonal temperature increased. The predominant AG recovered at this time was AG-G. Although the data are suggestive of a seasonal influence on plant infection, due to the small sample size at some collection times a more systematic sampling is needed before conclusions can be drawn.

One reason for observing a correlation between seasonal temperature changes and recovery of different AGs from strawberry roots in Connecticut is that there was a differential effect of temperature on virulence of the different AGs, demonstrated in growthchamber trials. Martin (12) observed little disease by any of the three AGs at $25^{\circ} \mathrm{C}$ but, at $15^{\circ} \mathrm{C}$, AG-I isolates were more virulent than AG-A or -G isolates. LaMondia and Martin (8) subsequently reported that $\mathrm{AG}-\mathrm{I}$ was more virulent at $10^{\circ} \mathrm{C}$ than $\mathrm{AG}-\mathrm{A}$ or $-\mathrm{G}$ but that, at $24^{\circ} \mathrm{C}, \mathrm{AG}-\mathrm{G}$ was more virulent. In contrast, Elmer and LaMondia (5) observed that AG-A was more virulent than AG-I or $-\mathrm{G}$ at $15^{\circ} \mathrm{C}$. In the initial description of $R$. fragariae, Husain and McKeen (7) reported that root infection was common at 5 to $10^{\circ} \mathrm{C}$ but was rare at 20 to $30^{\circ} \mathrm{C}$. Based on analysis of the isolate they deposited in the American Type Culture Collection (ATCC 14691; Manassas, VA), it was believed that their test was done with AG-A (12). It is possible that variation in isolate virulence may be the cause of some of these conflicting reports on the effect of temperature on virulence of a specific AG. The influence of temperature on virulence or recoverability of binucleate Rhizoctonia isolates from the coastal central California strawberry-production area has yet to be determined.

At the temperature regimes used in this experiment, there was no association between virulence and AG; all three AGs contained isolates with the same range of effects on plant growth parameters. However, differences in virulence within an AG were observed. For example, 98sOrt 1 and 98sOrt 12 are in AG-A and caused greater reductions in shoot and root biomass than the other AG-A isolates evaluated (Fig. 3). Similar results also were observed for plant mortality, with AG-I isolates 98Miller 6 and SpF24 causing plant mortality, whereas 98sOrt 21 did not. These observations on different levels of virulence of isolates from the same AG are similar to the results reported by Martin (12) for AGs-A and -I recovered from strawberry in Connecticut. Ribeiro and Black (16) also reported variation in virulence among binucleate isolates from strawberry; however, these isolates were not characterized to AG.

The standard binucleate AG tester isolates of Ogoshi et al. (14) did not provide a consistent assessment or accurate determination of AG for all isolates examined in this investigation. Some of the strawberry isolates did not anastomose with these tester isolates, even though the molecular data suggested that they were in the same AG. Subsequent investigations with different strawberry isolates of the same AG and RFLP group indicated there could be a

TABLE 2. Anastomosis grouping (AG), restriction fragment length polymorphism (RFLP) grouping, and sizes of initial amplified fragments and digested fragments after incubation with a restriction enzyme

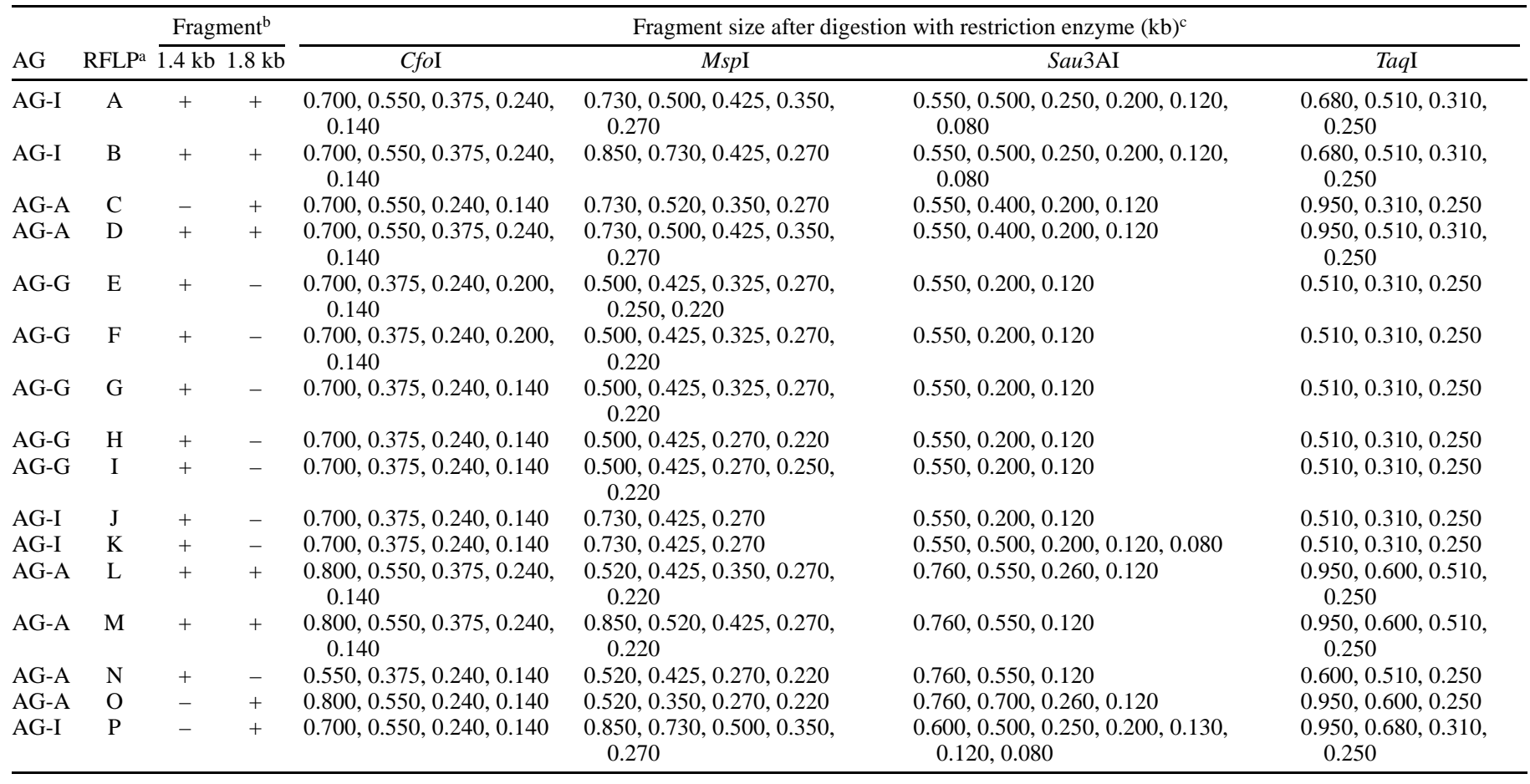

${ }^{a}$ RFLP groupings correspond to those shown in Figure 4.

${ }^{\mathrm{b}}$ Size of initial polymerase chain reaction amplified fragment; $+=$ presence and $-=$ absence.

${ }^{c}$ All estimates of fragment sizes were determined by comparison with 25- and 100-bp ladders, with measurements rounded to the nearest 5 bp. This was done for purposes of comparison among isolates; the values do not reflect absolute base pair fragment sizes. 
range in anastomosis reactions even within a group of similar isolates. Although there were strong anastomosis reactions among some isolates, for other pairings no anastomosis was observed. Variation among isolates in anastomosis reaction has been observed previously in Rhizoctonia spp. Ogoshi et al. (15) reported that paired binucleate isolates in the same AG exhibited both perfect and imperfect fusions (fused cells did not or did die, respectively). Furthermore, in some AGs (such as AGs-Ba and -Bb) there were distinct subgroups of isolates that had a higher frequency of anastomosis when paired with other isolates in the same subgroup

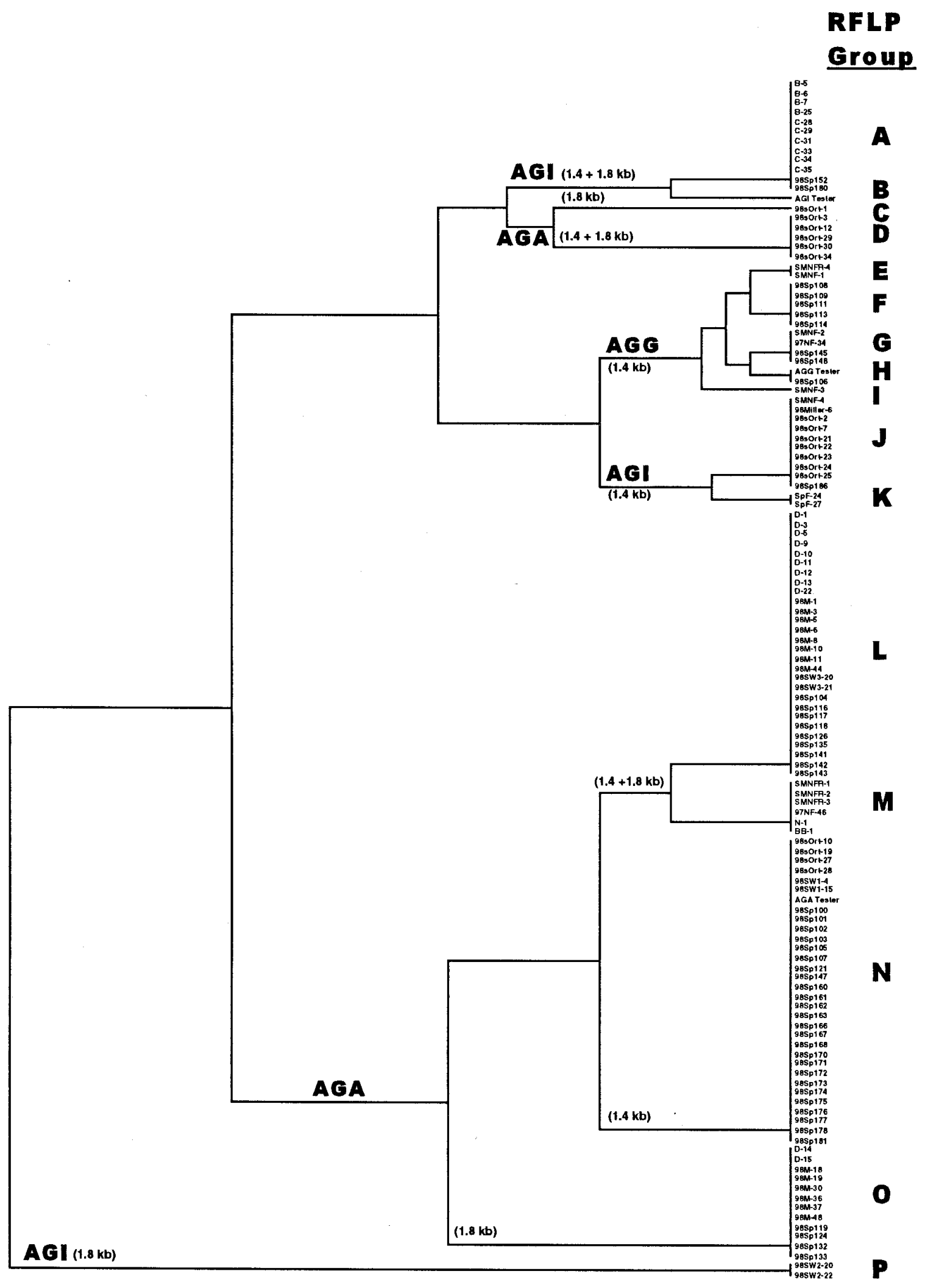

Fig. 4. Cluster analysis by unweighted pair group method using arithmetic averages of fragment sizes of an amplified region of the $25 \mathrm{~S}$ rDNA undigested and digested individually with four restriction enzymes (37 total character states). The anastomosis group (AG) and sizes of the initial amplified products are noted at the branches, and restriction fragment length polymorphism groupings are indicated on the right. 
compared with between subgroups. More extensive analysis of different types of anastomosis reactions has been done for the multinucleate $R$. solani, with the different types of reactions placed in four classifications (3). Reactions were classified as: no anastomosis = $\mathrm{C} 0$; and reactions with contact but no fusion, anastomosis of cells followed by cell death, or cell fusion with no cell death $=$ $\mathrm{C} 1, \mathrm{C} 2$, and $\mathrm{C} 3$, respectively. C3-type reactions are considered to be between hyphae that are closely related or from the same clonal lineage. The observed variations in anastomosis reactions with California strawberry isolates underscore the need to use multiple tester isolates in pairings to determine AG, as well as the benefit of using molecular techniques, such as the RFLP analysis described by Cubeta et al. (4), for isolate identification. A more detailed quantitative analysis of the anastomosis reaction among these isolates from California is needed to clarify the relationship among isolates in the same AG and RFLP group.

The technique described by Cubeta et al. (4) for identification of AGs of binucleate Rhizoctonia spp., using RFLP analysis of a PCR-amplified region of the rDNA, was effective for characterization of isolates recovered from strawberry. No single restriction enzyme accurately identified all AGs, but the use of a combination of the initial amplified fragment sizes and four restriction enzymes with UPGMA cluster analysis accurately grouped isolates to AG. Each AG had isolates in multiple RFLP groups; however, any one individual RFLP group contained isolates of only a single AG. The biological significance of the different RFLP groupings has yet to be determined. Different levels of anastomosis reactions (including lack of anastomosis) were observed among isolates of the same RFLP group; therefore, isolates in the same RFLP group do not reflect a single clonal isolate of the pathogen. There was no consistent correlation between RFLP group and the location of isolate collection or virulence on strawberry, although there was some indication that certain RFLP groups may be more virulent than others. For example, RFLP groups C and D (AG-A isolates that clustered separately from the other AG-A isolates) were more virulent than the AG-A isolates in RFLP groups L, M, N, and O. However, additional virulence testing with a greater number of isolates in each RFLP group is needed before conclusions about the relationship of RFLP grouping and virulence can be determined.

The strawberry isolates used in this analysis exhibited more variation in RFLP markers than in other reports on the use of this marker system for identification of binucleate Rhizoctonia spp. In the initial report on the use of this technique, Cubeta et al. (4) observed that AG-A, -G, and -I isolates from their investigation had amplified fragment sizes of $1.4,1.4$, and $1.4+1.8 \mathrm{~kb}$, respectively. The results with the strawberry isolates were similar, with the, exception that the AG-A isolates also had amplified fragment sizes of 1.8 or $1.4+1.8 \mathrm{~kb}$ and the AG-I isolates also had amplified fragment sizes of 1.4 or $1.8 \mathrm{~kb}$. Similar to the observations of Cubeta et al. (4), for some AG-I strawberry isolates there also was a 1.6-kb band occasionally amplified that was fainter than the 1.4- or 1.8-kb bands. This fainter band also was observed occasionally in strawberry AG-A isolates. This band was not consistently amplified in all PCR reactions, and because it was fainter than the other amplified bands, the digested products also were fainter and could be excluded from the data analysis.

When evaluating the intra-anastomosis group variation in restriction patterns, Cubeta et al. (4) observed that all AG-G isolates had the same RFLP group. In contrast, AG-G isolates recovered from strawberry were found in five RFLP groups with one polymorphic band observed for CfoI and two for MspI (Table 2). Likewise, the AG-A isolates investigated by Cubeta et al. (4) were reported to have only minor variations (usually one band), whereas the California strawberry isolates were more polymorphic, with isolates grouped on two separate clades. Part of the reason for the observed polymorphism is that AG-A strawberry isolates had a total of three banding patterns for the initial amplified fragment
$(1.4,1.8$, or $1.4+1.8 \mathrm{~kb})$ compared with a single $1.4-\mathrm{kb}$ band in the previously examined isolates (4). This may explain the separate clustering of isolates in RFLP groups $\mathrm{L}, \mathrm{N}$, and $\mathrm{O}$, but it does not account for the separation of isolates in RFLP groups L and M or the separation of these RFLP groups from RFLP groups C and D. These observations reflect the sequence variations inherent in the AG. Similar results also were observed for AG-I, in which the separation of RFLP group A or B $(1.4+1.8 \mathrm{~kb})$ from $\mathrm{J}$ or K $(1.4 \mathrm{~kb})$ was primarily due to new fragment sizes associated with the $1.8-\mathrm{kb}$ fragment of RFLP group A or B. However, RFLP group P (1.8 kb) was different from the other AG-I RFLP groups. Mazzola (13) also observed variation in the restriction banding profiles of AGs-I, -J, and -Q binucleate Rhizoctonia spp. isolates recovered from apple compared with the isolates examined by Cubeta et al. (4). In fact, the HpaII banding profile for the polymorphic AG-I isolates from apple was the same as the MspI (an isoschizemer of HpaII) restriction profile for RFLP group A from strawberry.

Although this RFLP marker system is effective for AG determinations of strawberry isolates, there are several considerations to keep in mind for validation of the technique. Due to a greater variation in RFLP grouping among isolates than previously observed $(4,13)$, it may be necessary to survey a number of isolates from a particular collection location to confirm the correlation between RFLP group and AG. Because an individual RFLP group represented only a single AG, the results of the UPGMA analysis can be used as a preliminary screen for selection of isolates for pairing with known AG tester isolates. Also, in some instances, specific bands of digested DNA may be fainter than others, thereby complicating data analysis if they are not taken into consideration. This was particularly evident in the current study for the $0.26-\mathrm{kb}$ Sau3A1 band of RFLP groups L and O, which was fainter than the other bands in the digest. One possible reason for these faint bands could be attributed to the binucleate nature of the isolates. It is quite possible that each of the nuclei have similarities, as well as differences, in restriction sites in the region of the rDNA that was examined. When restriction sites are conserved between the two nuclei, a single band will be observed but when polymorphic sites are present, a unique fragment size will be produced that is half the intensity of the bands generated from conserved restriction sites. This difference in band intensity would be further exacerbated if there were different copy numbers of the rDNA repeat unit in each nucleus as well. This explanation also would account for the observation that some isolates have two distinct amplified band sizes and that summation of band sizes can be larger than the initial sizes of the amplified fragments. Although it is possible that some of the fainter bands may be partial digests, the fact that the bands were consistently present in digestions of different amplification reactions with an excess of restriction enzyme argues against this.

Although the RFLP technique is useful for AG determinations of field isolates, additional research is needed before this marker system can be used to more fully evaluate the association of the RFLP grouping with particular traits and the population biology of the pathogen. Specifically, it is important to understand how the markers segregate in single basidiospore progeny. It was suggested earlier that dikaryotic hyphae could account for the two band sizes amplified in some isolates, as well as the faint bands observed after digestion of other isolates; this observation needs to be confirmed experimentally. Data on the behavior of single basidiospore progeny in the soil also is needed. Do they fuse with other single basidiospore isolates to form dikaryotic hyphae, or are they stable in the soil as monokaryotic isolates? Furthermore, what effect does this have on RFLP groupings and virulence of isolates? Once these questions have been addressed, additional experimentation exploring the relationship between RFLP group and virulence, as well as the recovery of specific RFLP groups at different times of the year, could be pursued.

The common recovery of binucleate Rhizoctonia spp. from necrotic roots of plants grown in nonfumigated soil and the virulence 
that many of these isolates exhibit in pathogenicity trials confirms the involvement of these pathogens in the strawberry root rot complex in California. Other fungal pathogens, such as Pythium and Cylindrocarpon spp., also were recovered frequently from these roots (F. N. Martin, unpublished data), but their effect on the host and possible interactions with binucleate Rhizoctonia spp. on the severity of root rot are unknown. However, in view of their level of recovery from the roots after the first 4 to 5 months after transplanting, it is believed that all three genera contribute to the significant reductions in plant growth observed when strawberry is grown in nonfumigated soil. At both the Watsonville and Salinas sampling locations, plant growth in test plots exhibited a 10 to $15 \%$ reduction in shoot growth within 8 weeks of transplanting into nonfumigated soil, compared with adjacent fumigated control plots (F. N. Martin, unpublished data). The root systems of these plants in nonfumigated soil also were less well developed when compared with plants in fumigated soil. This translates into a smaller, less healthy plant during the early spring that is unable to support the fruiting level expected for plants in an economically viable production field. Additional research is needed to determine the relative contribution of each fungal pathogen to the severity of root rot, the influence of seasonal variation in environmental conditions on relative infection frequencies, and the possible interactions among the different pathogens that influence root rot severity and its concomitant effect on yield reductions.

\section{ACKNOWLEDGMENTS}

Mention of trade names or commercial products in this manuscript is solely for the purpose of providing specific information and does not imply recommendation or endorsement by the United States Department of Agriculture. I thank C. Blackford, T. Price, and M. Orozco for their help in conducting portions of this research; M. Cubeta for useful discussions; K. Subbarao and D. Mitchell for their comments on the manuscript prior to submission; B. Mackey for assistance with SAS analysis; and F. Westerlund for providing plant samples from Santa Maria.

\section{LITERATURE CITED}

1. Anonymous. 1998. Agricultural Statistics. U.S. Government Printing Office, Washington, D.C.

2. Burpee, L. L., Sanders, P. L., Cole, H., Jr., and Sherwood, R. T. 1980. Anastomosis groups among isolates of Cerratobasidium cornigerum and related fungi. Mycologia 72:689-701.

3. Carling, D. E. 1996. Grouping in Rhizoctonia solani by hyphal anastomosis interactions. Pages 35-47 in: Rhizoctonia Species: Taxonomy, Molecular Biology, Ecology, Pathology, and Disease Control. B. Sneh, S. Jabaji-Hare, S. Neate, and G. Dijst, eds. Kluwer Academic Publishers, Dordrecht, Netherlands.

4. Cubeta, M. A., Echandi, E., Abernathy, T., and Vilgalys, R. 1991. Characterization of anastomosis groups of binucleate Rhizoctonia species using restriction analysis of an amplified ribosomal RNA gene. Phytopathology 81:1395-1400.

5. Elmer, W. H., and LaMondia, J. A. 1994. Chitosan inhibits Rhizoctonia fragariae but not strawberry black root rot. Adv. Strawberry Res. 13:26-31.

6. Himelrick, D. G., and Dozier, W. A. 1991. Soil fumigation and soil solarization in strawberry production. Adv. Strawberry Prod. 10:12-28.

7. Husain, S. S., and McKeen, W. E. 1963. Rhizoctonia fragariae sp. nov. in relation to strawberry degeneration in Southwest Ontario. Phytopathology 53:532-540.

8. LaMonda, J. A., and Martin, S. B. 1989. The influence of Pratylenchous penetrans and temperature on black root rot of strawberry by binucleate Rhizoctonia spp. Plant Dis. 73:107-110.

9. Larson, K. D., and Shaw, D. V. 1995. Relative performance of strawberry genotypes on fumigated and nonfumigated soils. J. Am. Soc. Hortic. Sci. 120:274-277.

10. Martin, F. N., and Semer, C. R. 1997. Selection of drug-tolerant strains of Pythium sylvaticum using sublethal enrichment. Phytopathology 87:685-692.

11. Martin, S. B. 1987. Rapid tentative identification of Rhizoctonia spp. associated with diseased turfgrasses. Plant Dis. 71:47-49.

12. Martin, S. B. 1988. Identification, isolation frequency, and pathogenicity of anastomosis groups of binucleate Rhizoctonia spp. from strawberry roots. Phytopathology 78:379-384.

13. Mazzola, M. 1997. Identification and pathogenicity of Rhizoctonia spp. isolated from apple roots and orchard soil. Phytopathology 87:582-587.

14. Ogoshi, A., Oniki, M., Araki, T., and Ui, T. 1983. Anastomosis groups of binucleate Rhizoctonia in Japan and North America and their perfect states. Trans. Mycol. Soc. Jpn. 24:79-87.

15. Ogoshi, A., Oniki, M., Sakai, R., and Ui, T. 1979. Anastomosis grouping among isolates of binucleate Rhizoctonia. Trans. Mycol. Soc. Jpn. 20:33-39.

16. Ribeiro, O. K., and Black, L. L. 1971. Rhizoctonia fragariae: A mycorrhizal and pathogenic fungus of strawberry plants. Plant Dis. Rep. 55:599-603.

17. Sambrook, J., Fritsch, E. F., and Maniatis, T. 1989. Molecular Cloning: A Laboratory Manual. 2nd ed. Cold Spring Harbor Laboratory Press, Cold Spring Harbor, NY.

18. Shaw, D. V., and Larson, K. D. 1996. Relative performance of strawberry cultivars from California and other North American sources in fumigated and nonfumigated soils. J. Am. Soc. Hortic. Sci. 12:764-767.

19. Sneh, B., Burpee, L., and Ogoshi, A. 1991. Identification of Rhizoctonia species. The American Phytopathological Society, St. Paul, MN.

20. Voth, V., and Bringhurst, R. S. 1990. Culture and physiological manipulation of California strawberries. HortScience 25:889-892.

21. Wilhelm, S. 1965. Pythium ultimum and the soil fumigation growth response. Phytopathology 55:1016-1020.

22. Wilhelm, S., Nelson, P. E., Thomas, H. E., and Johnson, H. 1972. Pathology of strawberry root rot caused by Ceratobasidium species. Phytopathology 62:700-705.

23. Wilhelm, S., and Paulus, A. O. 1980. How soil fumigation benefits the California Strawberry industry. Plant Dis. 64:264-270.

24. Wilhelm, S., Storkan, R. C., and Wilhelm, J. M. 1974. Preplant soil fumigation with methyl bromide-chloropicrin mixtures for control of soilborne diseases of strawberries-A summary of fifteen years of development. Agric. Environ. 1:227-236.

25. Wing, K. B., Pritts, M. P., and Wilcox, W. F. 1994. Strawberry black root rot: A review. Adv. Strawberry Res. 13:13-19.

26. Yuen, G. Y., Schroth, M. N., Weinhold, A. R., and Hancock, J.G. 1991. Effects of soil fumigation with methyl bromide and chloropicrin on root health and yield of strawberry. Plant Dis. 75:416-420. 